\title{
Habilidades Iniciais de Alfabetização em Português: Pesquisa Transcultural em Portugal e no Brasil
}

\author{
Ana Albuquerque - ISPA - Instituto Universitário, Lisboa, Portugal \\ Margarida Alves Martins - ISPA - Instituto Universitário, Lisboa, Portugal
}

\begin{abstract}
Resumo
O principal objetivo deste estudo foi avaliar os efeitos de um programa de treino de escrita inventada na aprendizagem de habilidades iniciais de alfabetização em crianças pré-escolares falantes de língua portuguesa. Participaram 79 crianças de educação infantil em Portugal e no Brasil. Constituíram-se quatro grupos (um grupo experimental e um grupo controle em cada país) equivalentes no conhecimento do alfabeto, inteligência não verbal e consciência fonológica (silábica e fonêmica). Foi realizado um pré/pós-teste de escrita e leitura. No período intermédio, os grupos experimentais participaram de atividades de escrita inventada e os grupos controle na leitura de livros infantis. No pós-teste, os resultados dos grupos experimentais foram superiores aos dos grupos controle nas tarefas de escrita e leitura. Além disso, os resultados foram equivalentes nos dois países, o que sugere que as atividades de escrita inventada promovem habilidades de alfabetização de pré-escolares falantes de duas variantes do português: europeu e brasileiro.

Palavras-chave: linguagem escrita, leitura, habilidades metalinguísticas, alfabetização, educação infantil
\end{abstract}

Early Literacy Skills in Portuguese: a Crosscultural Research in Portugal and Brazil

\begin{abstract}
The main goal of this study was to assess the effects of an invented spelling training programme in the early stages of literacy of preschool Portuguese-speaking children. It encompassed 79 children from Portugal and Brazil. There were four equivalent groups (one experimental group and one control group in each country) and various control measures: knowledge of the alphabet, non-verbal intelligence and phonological awareness (syllabic and phonemic). A reading and spelling pre/post-test was carried out. During the intermediate period the experimental groups participated in invented spelling activities, and the control groups read children's books. The results of the post-test have shown that the experimental groups reached higher levels in both reading and spelling as compared to the control groups. This evolution pattern was found in both countries, thus suggesting that invented spelling activities promote early literacy skills of preschool children speaking two Portuguese variants: European and Brazilian.

Keywords: written language; reading; metalinguistic skills; literacy; early childhood education
\end{abstract}

Habilidades de Alfabetización Temprana en Portugués: Investigación Multicultural en Portugal y Brasil

\begin{abstract}
Resumen
El principal objetivo de este estudio fue evaluar los efectos de un programa de entrenamiento de escritura inventada, en el aprendizaje de habilidades iniciales de alfabetización, en niños preescolares que hablan lengua portuguesa. Participaron 79 niños de educación infantil en Portugal y Brasil. Se constituyeron cuatro grupos (un grupo experimental y un grupo control en cada país) equivalentes en conocimiento de alfabeto, inteligencia no verbal y conciencia fonológica (silábica y fonémica). Fue realizado un pre/post-test de escritura y lectura. En el período intermedio, los grupos experimentales participaron en actividades de escritura inventada y los grupos de control en la lectura de libros infantiles. En el post-test, los grupos experimentales tuvieron resultados superiores a los grupos de control, en lo que se refiere a tareas de escritura y lectura. Además, los resultados fueron equivalentes en los dos países, lo que sugiere que las actividades de escritura inventada promueven habilidades de alfabetización de preescolares que hablan las dos variantes de portugués: europeo y brasileño.

Palabras-clave: lenguage escrito; lectura; habilidades metalingales; alfabetización; educación infantil
\end{abstract}

\section{Introdução}

As habilidades de leitura e escrita são uma aprendizagem essencial para o sucesso no percurso escolar das crianças. Atualmente, vivemos numa sociedade repleta de linguagem escrita, o que constitui um ambiente estimulante para a criança, que vai estabelecendo contato e interações com material escrito no seu cotidiano. Os trabalhos pioneiros de Chomsky (1970), Read (1971) e
Ferreiro e Teberosky (1979) mostraram que as crianças pequenas são capazes de adquirir conhecimentos de leitura e escrita antes do ensino escolar formal. Apesar da exposição diária aos estímulos não ser suficiente para o domínio da alfabetização, que requer o ensino direto de aspectos específicos da língua, o universo de escrita potencializa a aquisição de habilidades metalinguísticas.

Segundo Gombert (1990), as habilidades metalinguísticas compreendem a reflexão sobre a 
linguagem e a sua utilização, e o controle e planificação dos próprios processos de tratamento linguístico, tanto em situações de compreensão da linguagem, como em situações de produção da linguagem. Uma dessas habilidades é a consciência fonológica. Tratase, segundo esse autor, da capacidade para identificar as componentes fonológicas das unidades linguísticas (sílabas, unidades intrassilábicas e fonemas) e de as manipular de uma forma deliberada. Nunes e Bryant (2009) consideram que o desenvolvimento dessa consciência é essencial para a compreensão e interiorização do princípio alfabético.

A tarefa de aprender a ler e escrever implica uma reflexão consciente e explícita acerca da linguagem oral e escrita (Snow, 1991; Teale \& Sulzby, 1986). Com efeito, para que a aprendizagem da leitura se realize com sucesso, é imperativo saber analisar a estrutura fonêmica da fala e compreender que os grafemas representam um sistema de notação de sons (Ehri, 2014; Gombert, 1990; Mann \& Foy, 2003; Morais, 2013).

O conhecimento das letras do alfabeto é também essencial, pois fornece uma representação visual dos fonemas da linguagem oral (Boyer \& Ehry, 2011; Cardoso-Martins, Mesquita, \& Ehri, 2011; Cardoso-Martins, Resende, \& Rodrigues, 2002; Castiglioni-Spalten \& Ehri, 2003; Stahl \& Murray, 1998). A compreensão das relações entre linguagem oral e linguagem escrita é facilitada pelo fato de grande parte das letras conter no seu nome pelo menos um dos sons que representa (Pollo, Kessler, \& Treiman, 2005; Treiman, Tincoff, Rodriguez, Mozaki, \& Francis, 1998).

Diversas pesquisas têm mostrado que a conjugação dos conhecimentos fonológicos com o conhecimento do nome das letras é uma base sólida para a compreensão do sistema alfabético de escrita (Cardoso-Martins, 1995; Pollo, Treiman, \& Kessler, 2008).

Byrne (1998) argumenta que as crianças podem ter uma noção adequada da estrutura fonêmica do discurso oral sem entender necessariamente a natureza alfabética da linguagem escrita. Essa compreensão requer que a criança possua não somente competências de análise fonêmica, mas que seja capaz de a coordenar com as letras. É essa coordenação que permite a descoberta do princípio alfabético.

Diversos pesquisadores (Alves Martins, Albuquerque, Salvador, \& Silva, 2013; Chomsky, 1970; Ferreiro \& Teberosky, 1979; Ouellette \& Sénéchal, 2008a, b; Read, 1971; Sénéchal, LeFevre, Smith-Chant, \& Colton, 2001) sugerem que o processo de aquisição da linguagem escrita surge antes da escolarização. Com efeito, a interação com a escrita desde cedo, pormeio de jogos e brincadeiras com a linguagem, as letras e os sons, as tentativas de escrita e as tentativas de leitura, promove o conhecimento de unidades visuais e fonológicas úteis para a alfabetização (Dehaene, 2012; Tolchinsky, 2005).

Sénéchal, LeFevre, Smith-Chant e Colton (2001) chamaram a atenção para o estudo sistemático da forma como esses diferentes estímulos podem facilitar a aquisição de princípios associados à leitura e escrita. Vários estudos demonstraram que as escritas precoces, também designadas de escritas inventadas, são um fator importante para a aprendizagem, pois não se tratam unicamente de processos de cópia ou memorização (Adams, 1998; Mann, 1993; McBride-Chang, 1998; Ouellette \& Sénéchal, 2008a, b; Tolchinsky, 2005; Treiman, 1998), uma vez que refletem mecanismos de experimentação e reflexão em que as crianças usam os seus recursos cognitivos e memória efetiva para atribuírem um sentido ao escrito.

Embora as escritas iniciais possam ter poucas semelhanças com a escrita convencional, com o tempo é notória uma progressão na sua acuidade fonológica e complexidade ortográfica (Ouellette \& Sénéchal, 2008a; Treiman \& Kessler, 2003). Também Ehri (1991) considera que numa primeira fase de desenvolvimento da linguagem escrita (fase pré-alfabética), as crianças combinam letras e pseudoletras de forma arbitrária sem prestar atenção às relações entre letras e sons; numa segunda fase (alfabética parcial), as crianças começam a representar alguns dos sons e sílabas das palavras usando letras que conhecem; na terceira fase (alfabética) existe uma correspondência sistemática entre letras e sons, embora sem respeito pelas convenções ortográficas; na fase final (alfabética consolidada), as crianças têm também em conta fatores morfêmicos, sendo já considerados leitores/escritores proficientes.

Bowman e Treiman (2002) afirmam que "a escrita pode encorajar as crianças a usar o princípio alfabético de uma forma que a leitura não o faz e pode ensinar habilidades que poderão ser transferidas para a leitura" (p. 31). Nesse sentido, as atividades promotoras de escrita inventada nos anos pré-escolares são uma ferramenta útil, pois encorajam a reflexão sobre os constituintes do oral, as marcas gráficas e as associações entre ambas (Alves Martins \& Silva, 2006a, b; Ouellette \& Sénéchal, 2008a; Ouellette, Sénéchal, \& Haley, 2013; Silva \& Alves Martins, 2002; Treiman, 2004).

Desse modo, têm sido criados programas de treino para crianças de educação infantil no sentido de 
promover as escritas inventadas por meio da reflexão sobre as letras e os sons de determinadas palavras simples. Esses programas, nomeadamente realizados em inglês (Ouellette \& Sénéchal, 2008b; Sénéchal, Ouellette, Pagan, \& Lever, 2012) e em português europeu (Alves Martins, Albuquerque, Salvador, \& Silva, 2013, 2015; Alves Martins, Salvador, Albuquerque, \& Silva, 2014; Alves Martins \& Silva, 2006a, b; Silva \& Alves Martins, 2002), têm demonstrado efeitos benéficos no desenvolvimento da escrita e da consciência fonêmica.

Um estudo experimental em inglês desenvolvido no Canadá por Ouellette e Sénéchal (2008b) procurou testar a relação entre escrita inventada e aprendizagem da leitura com três grupos de crianças de cinco anos ao longo de nove sessões de intervenção. No grupo de escrita inventada, a tarefa das crianças consistia em escrever um conjunto de palavras da melhor forma que fossem capazes, sendo apresentada posteriormente a mesma palavra com uma ou mais letras corretas (feedback desenvolvimental adequado). Os grupos de comparação participaram de um treino de consciência fonológica ou atividades de desenho. Os resultados desse estudo mostraram uma interação causal e o impacto positivo da escrita inventada com feedback adequado para as habilidades de escrita, consciência fonológica, conhecimento ortográfico e leitura de palavras.

Uma pesquisa realizada posteriormente (Sénéchal et al, 2012) com crianças com fracas habilidades fonológicas obteve dados semelhantes. Os resultados encontrados sugerem o efeito preventivo desses programas de treino de escrita inventada quanto a futuras dificuldades na aquisição da leitura.

Estudos experimentais foram também realizados por pesquisadores portugueses (Alves Martins et al., 2013, 2015; Alves Martins et al., 2014; Alves Martins \& Silva, 2006a, b; Silva \& Alves Martins, 2002), que efetuaram programas de treino, convidando crianças pré-escolares para participar de breves discussões sobre a escrita de palavras simples mediante o monitoramento do adulto, que promovia a reflexão acerca das letras e sons. Em Portugal, têm sido obtidos resultados bastante positivos em contexto individual e de pequeno grupo, melhorando a qualidade das escritas iniciais, a consciência fonêmica e a leitura de crianças pré-escolares falantes de português europeu.

Desse modo, torna-se interessante desenvolver esses programas com crianças da mesma faixa etária falantes de português brasileiro, para compreender se os efeitos podem ser alargados a outra variante linguística do mesmo idioma (Mateus et al., 2003). A língua portuguesa não é homogênea, como é o caso do português europeu (PE) e do português brasileiro (PB), que apresentam diferenças fonológicas, lexicais e sintáticas muito salientes (Cuesta \& Luz, 1971; Duarte, 2000).

Assim, o principal objetivo deste estudo é avaliar os efeitos de um programa de treino de escrita inventada na aprendizagem de habilidades iniciais de leitura e escrita em crianças pré-escolares falantes de língua portuguesa em Portugal e no Brasil.

\section{Método}

\section{Desenho Experimental}

O presente estudo é de natureza experimental com duas condições: experimental e controle. Foram constituídos dois grupos experimentais (GE) e dois grupos-controle (GC) em cada um dos dois países (Portugal e Brasil). As habilidades de leitura e escrita das crianças em educação infantil foram avaliadas em situação de pré-teste e pós-teste. No período de intervenção, as crianças dos grupos experimentais participaram de um programa de treino de escritas inventadas e as crianças dos grupos-controle realizaram atividades de leitura de livros infantis. O trabalho foi conduzido no ambiente escolar por um pesquisador/psicólogo treinado.

\section{Participantes}

Participaram deste estudo 79 crianças pré-escolares de nacionalidade portuguesa $(n=52)$ e brasileira $(n=27)$, selecionadas de quatro escolas de educação infantil: duas na cidade de Lisboa (Portugal) e duas na cidade de São Paulo (Brasil). Apenas foram incluídas na amostra as crianças autorizadas pelos responsáveis legais por meio do Termo de Consentimento Livre e Esclarecido. A língua materna das crianças era o português (PE e $\mathrm{PB}$, respetivamente) e não sabiam ler nem escrever, pois o currículo acadêmico na pré-escola nos dois países não inclui atividades de linguagem escrita até o começo da educação formal.

Em cada escola, foram selecionados aleatoriamente os participantes para as duas condições (experimental e controle). Utilizou-se como critério que o número de crianças da condição experimental fosse múltiplo de 4, para que os participantes dessa condição trabalhassem em pequenos grupos de quatro crianças.

Em Portugal, numa escola foram autorizadas 29 crianças $(G E=16 ; G C=13)$ e na outra, 23 crianças $(G E=12 ; G C=11)$; no Brasil, numa escola foram 
autorizadas 10 crianças $(G E=4 ; G C=6)$ e na outra, 17 crianças $(G E=8 ; G C=9)$. Em cada uma das escolas dos dois países, as crianças pertenciam à mesma turma.

A amostra final ficou constituída por 28 crianças em Portugal e 12 no Brasil nas condições experimentais, e 24 em Portugal e 15 no Brasil nas condições de controle. Posteriormente foram avaliados o conhecimento do alfabeto, a inteligência não verbal e a consciência fonológica (silábica e fonêmica) para controlar o efeito dessas variáveis.

\section{Instrumentos e Procedimentos}

\section{Conbecimento do Alfabeto}

Nessa tarefa, realizada individualmente, o pesquisador apresentou de forma aleatória um conjunto de 26 cartões com as letras do alfabeto impressas na forma maiúscula, sendo pedido às crianças para nomear cada uma. Contabilizou-se o número de letras identificadas corretamente, sendo possível obter um escore total de 26 pontos.

\section{Inteligência Não Verbal}

Foi utilizado o Teste Matrizes Progressivas de Raven (versão colorida) (Raven, Raven, \& Court, 1998) para avaliar as habilidades cognitivas de raciocínio não verbal, sendo pedido às crianças para identificar o elemento adequado em falta de um padrão incompleto. Cada resposta correta recebeu 1 ponto, num total de 0 a 36 pontos.

\section{Consciência Fonológica}

Nessa tarefa, optou-se por utilizar provas diferentes em cada país, considerando as diferenças no acento e entonação do PE e do PB, para controlar efeitos que pudessem interferir nos aspectos fonéticos do discurso. Selecionaram-se duas provas estandardizadas e padronizadas para a população, semelhantes quanto à natureza da tarefa proposta: em Portugal, a Bateria de Provas Fonológicas (Silva, 2008) e, no Brasil, o CONFIAS - Consciência Fonológica: Instrumento de Avaliação Sequencial (Moojen et al., 2003). Em cada prova, utilizou-se um subteste de identificação da sílaba inicial e do fonema inicial - as crianças deveriam escolher, dentre quatro palavras ditas pelo examinador e com suporte de imagens, duas com o mesmo som inicial (sílaba/fonema). As respostas corretas receberam 1 ponto, sendo o escore total máximo de 14 valores em cada subteste na prova portuguesa e de 4 valores na prova brasileira.

\section{Pré/Pós-Teste: Tarefa de Escrita}

O pré/pós-teste de escrita foi administrado de forma individual em duas sessões com duração aproximada de 15 minutos. Foi pedido às crianças que escrevessem, da melhor forma que fossem capazes, um conjunto de 18 palavras ditadas pelo examinador. Durante a atividade não foi dado nenhum tipo de feedback ou informação adicional, sendo as crianças encorajadas a escrever como soubessem. As palavras foram as mesmas nos dois países e tinham estruturas internas silábicas frequentes na língua portuguesa, sendo compostas por vogais abertas, consoantes oclusivas e fricativas. Contou-se o número de correspondências grafo-fonológicas adequadas em cada palavra.

\section{Pré/Pós-Teste: Tarefa de leitura}

O pré/pós-teste de leitura foi também administrado de forma individual em duas sessões e com uma semana de intervalo em relação ao pré/pós-teste de escrita. Nessa prova, apresentou-se um conjunto de 18 cartões com as mesmas palavras da tarefa de escrita, uma de cada vez em ordem aleatória fixa. A tarefa das crianças consistia em ler as palavras da melhor forma possível. Para a análise das leituras, contou-se o número de fonetizações adequadas em cada palavra lida a partir do registro áudio das respostas.

Programa dos Grupos Experimentais - Treino de Escritas Inventadas

O programa de treino de escritas inventadas teve início na semana seguinte ao pré-teste e foi constituído por 10 sessões bissemanais com duração aproximada de 20 minutos. As atividades centraram-se exclusivamente na componente escrita das palavras sem intervenção na leitura. Foram planejadas atividades de escrita inventada para encorajar os participantes a refletir sobre os grafemas e fonemas e promover a compreensão do princípio alfabético. Todas as atividades e a forma como foram conduzidas foram iguais ao longo das sessões.

Os participantes foram divididos em grupos de quatro. Procurou-se, sempre que possível, que cada grupo fosse constituído por crianças com diferentes níveis de consciência silábica e fonêmica e com diferentes conhecimentos sobre as letras.

Nas sessões de treino, foi pedido às crianças para discutir sobre a escrita de quatro palavras, uma de cada vez, e indicar as letras escolhidas, para que o examinador escrevesse numa folha em maiúsculas. No final de cada palavra, era mostrada ao grupo a mesma 
palavra escrita corretamente, sendo dito que tinha sido produzida por um grupo de crianças de outra escola. Era em seguida pedido para refletirem sobre as duas configurações da mesma palavra e decidir qual seria a mais correta.

O adulto encorajava a participação ativa das crianças nas discussões, atuando como mediador e facilitador das dinâmicas e interações do grupo e estimulando o pensamento sobre a codificação dos fonemas nas palavras. Assim, colocava questões sobre os grafemas e os fonemas presentes nas duas formas de escrita da mesma palavra, induzindo a reflexão sobre as relações oral-escrito.

As palavras utilizadas no treino foram selecionadas pelo examinador e eram diferentes das palavras do pré/pós-teste. Nas primeiras sessões, foram utilizadas palavras começadas com a mesma letra e, nas sessões seguintes, foi pedido às crianças que escrevessem palavras começadas com diferentes grafemas. Foi realizado o registro áudio das sessões para futura transcrição e análise das interações. Apresenta-se a seguir um exemplo ilustrativo das dinâmicas numa sessão de treino.

Pesq.: Hoje vamos escrever a palavra DATA. Qual será a primeira letra?

Catarina: $D, D, D$ !

Isabel: / da/, / da/, / da/... éum A!

Pesq.: A Catarina escuton o som do D primeiro. E vocês também escutaram?

Isabel: Eu não.

Pesq.: Pensem lentamente na palavra DATA. / da/-/ta/. Qual som vocês escutam?

Henrique: /da/, /da/... /da/, /da/.

Isabel: Agora eu consegui escutar o som do D!

Pesq.: Você escutou também? / da/.../də/. Então vamos escrever um D. [Escrita: D]

Isabel, Henrique, Catarina: Agora é um A!

Pesq.: Vou escrever um $A$ aqui? [Escrita: DA]. E agora?

Teresa: $T, T, T !$

Pesq.: Vocês concordam com a Teresa? Vocês acham que agora é a letra T?

Isabel, Henrique, Catarina: /to/, /to/... TA, TA, TA... isso, a última letra é o T!
Pesq.: [Escrita: DAT]. Agora eu vou mostrar para vocês como as crianças da outra escola escreveram DATA. Foi assim. Está igual ou está diferente?

Isabel: Oh! A gente deveria ter escrito um A aqui [apontando para a última letra].

Pesq.: Vamos pensar. Por que vocês acham que escreveram um $D$ primeiro?

Catarina: Porque é / da/.

Teresa: Isso, /da/, / da/... éD e depois um A!

Isabel: E se fosse um I era /di/-/ta/.

Pesq.: E a última letra, por que vocês acham que eles escreveram um $A$ no final?

Henrique: Ah, en sei! Porque é / da/-/ta/! / t $\alpha / \ldots / \alpha /$, $/ \alpha /, / \alpha /$ !

\section{Programa dos Grupos-Controle - Leitura de Livros Infantis}

As crianças dos grupos controle foram também divididas em pequenos grupos de três a quatro crianças e participaram de um programa semelhante ao programa do grupo experimental em frequência e duração. Nas sessões realizaram-se atividades de leitura de livros infantis.

\section{Resultados}

\section{Dados Preliminares}

Para assegurar a equivalência dos grupos no começo do estudo, foram realizados testes $t$ para amostras independentes, assumindo o grupo (experimental/ controle) como variável independente e as diversas medidas iniciais como variáveis dependentes: idade no início do ano letivo (em meses), conhecimento do alfabeto, inteligência não-verbal, consciência silábica e consciência fonêmica. As análises estatísticas foram realizadas separadamente para a amostra de cada país, sendo os resultados descritos na Tabela 1 .

As análises efetuadas indicaram que, no início da pesquisa, não havia diferenças estatisticamente significantes entre os grupos experimentais e controle nos dois países, obtendo-se os seguintes resultados na amostra portuguesa e brasileira, respetivamente: idade em meses, $t(50)=-1,48, p=0,14 ; t(25)=-1,34, p=$ 0,19 ; conhecimento do alfabeto, $t(50)=0,34, p=0,73$; $t(25)=-0,02, p=0,99$; inteligência não verbal, $t(50)=$ $-1,27, p=0,21 ; t(25)=0,27, p=0,78$; consciência silábica, $t(50)=-0,65, p=0,52 ; t(25)=0,38, p=0,71$; e 
Tabela 1

Médias e Desvios Padrões para as Medidas Iniciais Avaliadas

\begin{tabular}{|c|c|c|c|c|c|c|c|c|c|c|}
\hline & \multicolumn{2}{|c|}{ Idade meses } & \multicolumn{2}{|c|}{$\begin{array}{c}\text { Alfabeto } \mathrm{n}^{\circ} \\
\text { letras }\end{array}$} & \multicolumn{2}{|c|}{ Inteligência } & \multicolumn{2}{|c|}{ C. Silábica } & \multicolumn{2}{|c|}{ C. Fonêmica } \\
\hline & $M$ & $D P$ & M & $D P$ & $M$ & $D P$ & $M$ & $D P$ & $M$ & $D P$ \\
\hline GE_Portugal & 62,68 & 3,95 & 16,07 & 4,17 & 16,82 & 4,76 & 4,96 & 3,52 & 2,71 & 1,82 \\
\hline GC_Portugal & 61,21 & 3,05 & 16,46 & 3,90 & 15,21 & 4,33 & 4,33 & 3,49 & 2,96 & 1,85 \\
\hline GE_Brasil & 64,83 & 3,33 & 19,17 & 5,24 & 14,50 & 3,68 & 1,67 & 0,98 & 1,25 & 1,14 \\
\hline GC_Brasil & 63,13 & 3,23 & 19,13 & 4,63 & 15,00 & 5,09 & 1,80 & 0,86 & 1,47 & 0,99 \\
\hline
\end{tabular}
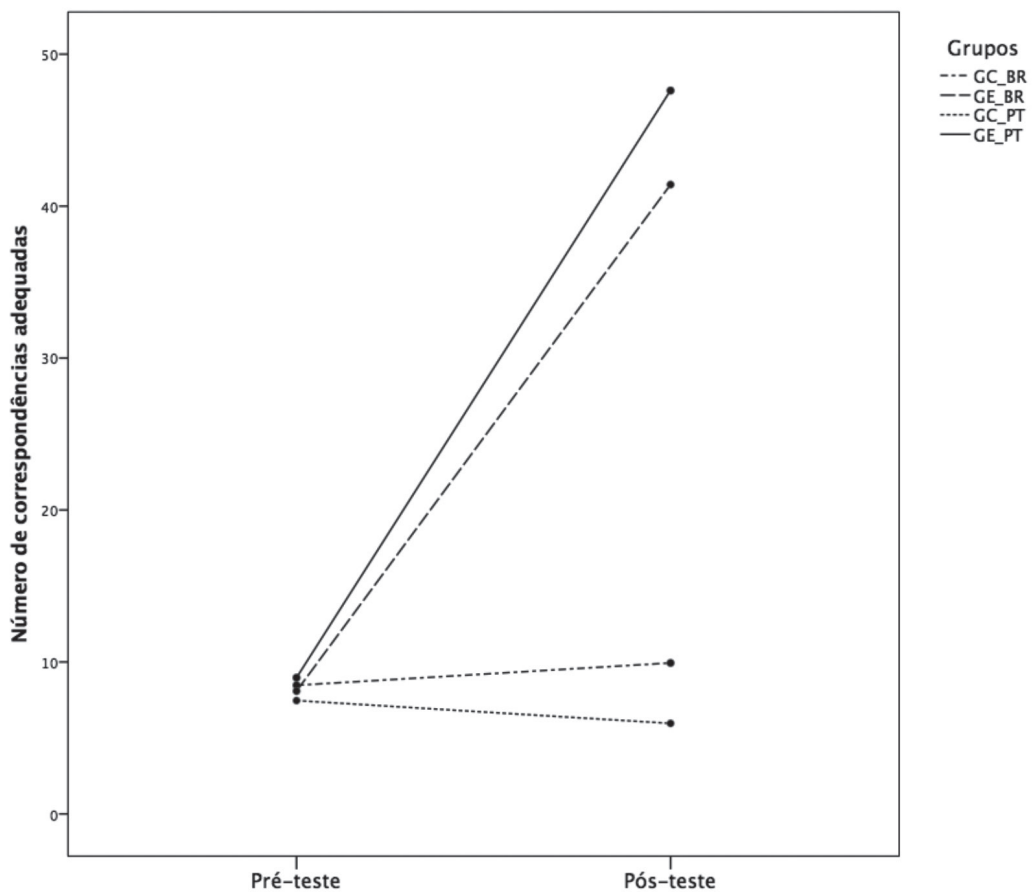

Figura 1. Número de correspondências adequadas na tarefa de escrita nos dois momentos de avaliação.

consciência fonêmica, $t(50)=0,48, p=0,64 ; t(25)=$ $0,53, p=0,60$.

\section{Desempenho na Tarefa de Escrita}

Para analisar as produções escritas das crianças na tarefa de escrita (pré/pós-teste), contou-se o número de correspondências grafo-fonológicas adequadas, isto é, os grafemas corretamente escritos em cada palavra. São apresentados na Figura 1 os escores médios para os grupos experimentais e para os grupos controle, separados por amostra de cada país, na avaliação de pré-teste e pós-teste.

Como é possível observar a partir do gráfico, os quatro grupos do estudo apresentavam resultados muito semelhantes no pré-teste de escrita. Na avaliação de pós-teste, foi notória uma grande evolução nos grupos experimentais, enquanto os grupos controle praticamente mantiveram os seus escores iniciais. Para analisar os resultados obtidos no pós-teste pelos grupos experimentais, tanto em Portugal como no Brasil, em relação aos escores obtidos pelos grupos controle, efetuou-se uma análise de covariância, tendo como variável independente os grupos, como covariável os resultados no pré-teste e, como variável dependente, os resultados no pós-teste. Os testes executados revelaram diferenças estatisticamente significantes entre os grupos $\left(F(3,74)=80,90, p<0,01, \eta_{p}^{2}=0,77\right)$. Uma análise post hoc com o teste Bonferroni confirmou 
que ambos os grupos experimentais tiveram melhores resultados do que os grupos controle $(p<0,01)$. Além disso, não foram encontradas diferenças estatisticamente significantes entre o grupo experimental em Portugal e o grupo experimental no Brasil $(p=0,831)$, nem entre os grupos controle nos dois países $(\phi=1,00$ para ambas as comparações). Para ilustrar essas diferenças, apresentam-se na Tabela 2 alguns exemplos reais de produções escritas no pré-teste e no pós-teste em três das palavras avaliadas.

Genericamente, os exemplos apresentados sugerem que, no pré-teste, as crianças adotavam estratégias de escrita sem critérios linguísticos, recorrendo a letras, pseudoletras e números de forma arbitrária e aleatória. Essa característica está presente nos dois grupos do estudo e é independente do país de proveniência dos participantes. No pós-teste, verificou-se que as crianças dos grupos experimentais codificaram adequadamente determinados segmentos das palavras, apresentando grafemas corretos para representar alguns fonemas das palavras, tanto na amostra portuguesa como brasileira. Não se observou essa evolução nos grupos controle, pois, de uma forma geral, as crianças mantiveram os mesmos critérios não linguísticos nas suas escritas.

\section{Desempenho na Tarefa de Leitura}

Para a análise das leituras das crianças, contou-se o número de fonetizações adequadas, isto é, o número de grafemas corretamente lidos em cada palavra. $\mathrm{Na}$ Figura 2, são descritas as médias dos grupos em cada país, no pré-teste e no pós-teste.

O gráfico demonstra como os grupos experimentais tiveram um progresso na leitura muito superior aos grupos-controle, independentemente do país da amostra. No início do estudo, os quatro grupos eram equivalentes, verificando-se uma grande diferença de resultados na avaliação de pós-teste entre as crianças que participaram e as que não participaram do programa de treino de escrita. Uma análise de covariância assumindo os grupos como variável independente, os resultados no pré-teste como covariável e os resultados no pósteste como variável dependente, evidenciou diferenças estatisticamente significantes entre os grupos $(F(3,74)$ $\left.=19,17, p<0,01, \eta_{p}^{2}=0,44\right)$. De acordo com o teste post hoc de Bonferroni verificaram-se diferenças entre os grupos experimentais em comparação com os grupos controle $(p<0,01$ para os grupos em Portugal, $p$ $<0,01$ para os grupos no Brasil). Além disso, não se verificaram diferenças estatisticamente significantes entre os grupos experimentais de Portugal e do Brasil nem entre os grupos controle de ambos os países $(p=$ 1,00 para ambas as comparações). Na Tabela 3, apresentam-se alguns exemplos das diferenças na leitura das crianças dos dois grupos, no pré-teste e no pós-teste, relativamente a três das palavras avaliadas.

Os exemplos expostos na Tabela 3 sugerem que, no pré-teste, as palavras foram lidas de forma totalmente aleatória e sem acesso a princípios linguísticos. No pós-teste, foram evidentes as diferenças entre as leituras das crianças dos grupos experimentais e dos grupos-controle. Após a participação do programa de treino de escritas inventadas, tanto os participantes portugueses como brasileiros manifestaram estratégias adequadas de decodificação de alguns fonemas, lendo corretamente determinados segmentos das palavras e, em alguns casos, inclusive as palavras na sua totalidade.

\section{Discussão}

O principal objetivo do presente estudo foi avaliar o impacto de um programa de treino de escritas inventadas na aprendizagem de habilidades iniciais de leitura e escrita em crianças pré-escolares falantes de língua portuguesa, em Portugal e no Brasil. Verificou-se que, após terem participado do programa de treino, as crianças dos grupos experimentais dos dois países

Tabela 2

Exemplos de Respostas das Crianças no Pré/Pós-Teste - Tarefa de Escrita

\begin{tabular}{|c|c|c|c|c|c|c|c|c|}
\hline \multirow{3}{*}{ Palavras } & \multicolumn{4}{|c|}{ Grupos Experimentais } & \multicolumn{4}{|c|}{ Grupos-Controle } \\
\hline & \multicolumn{2}{|c|}{ Amostra portuguesa } & \multicolumn{2}{|c|}{ Amostra brasileira } & \multicolumn{2}{|c|}{ Amostra portuguesa } & \multicolumn{2}{|c|}{ Amostra brasileira } \\
\hline & Pré-teste & Pós-teste & Pré-teste & Pós-teste & Pré-teste & Pós-teste & Pré-teste & Pós-teste \\
\hline BOTA & B & BOTA & UIRI & $\mathrm{OA}$ & POR & MVIEL & SAM & DEMAEF \\
\hline VAI & RREFLCO & VI & $\mathrm{RC}$ & VA & A & LT & MDA & EOO \\
\hline FADA & $\mathrm{VO}$ & FAD & A & AADA & RPIOA & RIMANB & $\mathrm{R} \# 2$ & EIU \\
\hline
\end{tabular}




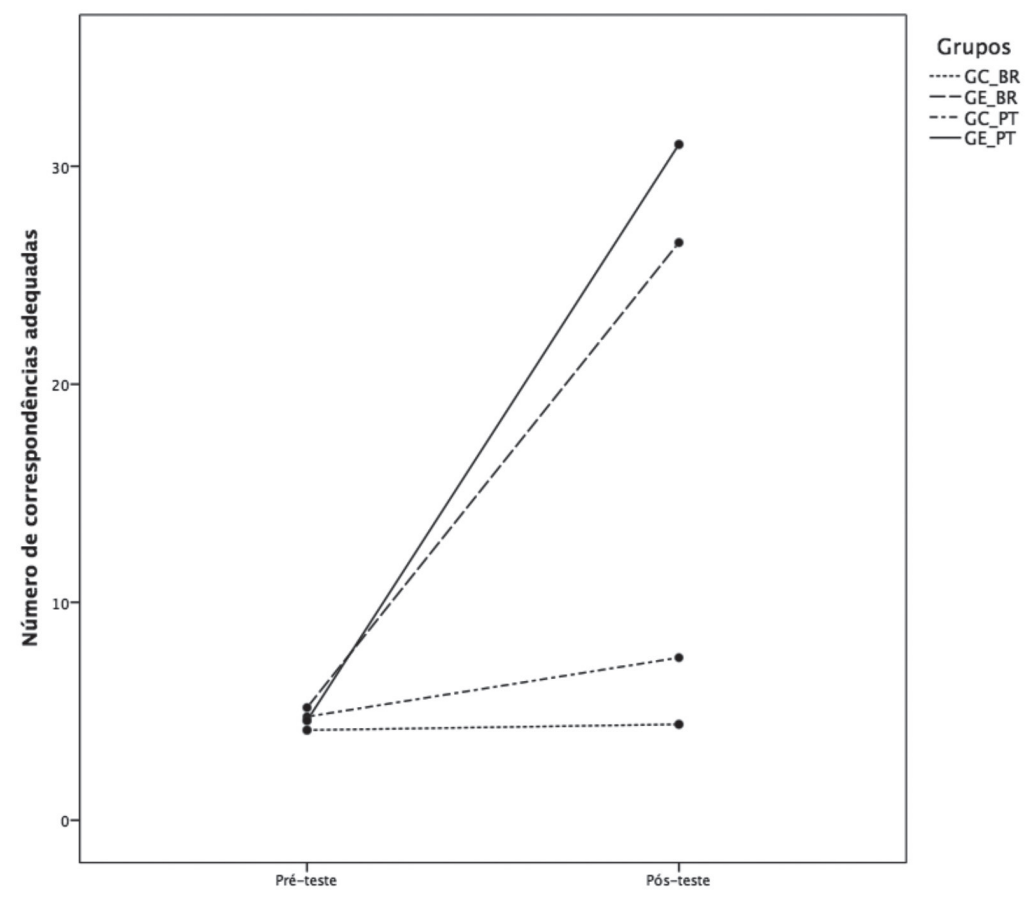

Figura 2. Número de correspondências adequadas na tarefa de leitura nos dois momentos de avaliação.

Tabela 3

Exemplos de Respostas das Crianças no Pré/Pós-Teste - Tarefa de Leitura

\begin{tabular}{|c|c|c|c|c|c|c|c|c|}
\hline \multirow{3}{*}{ Palavras } & \multicolumn{4}{|c|}{ Grupos Experimentais } & \multicolumn{4}{|c|}{ Grupos-Controle } \\
\hline & \multicolumn{2}{|c|}{ Amostra portuguesa } & \multicolumn{2}{|c|}{ Amostra brasileira } & \multicolumn{2}{|c|}{ Amostra portuguesa } & \multicolumn{2}{|c|}{ Amostra brasileira } \\
\hline & Pré-teste & Pós-teste & Pré-teste & Pós-teste & Pré-teste & Pós-teste & Pré-teste & Pós-teste \\
\hline BOTA & Tomás & Bota & Cristina & Bola & Futebol & Chapéu & Caneta & Pessoa \\
\hline VAI & Rua & Vati & Ovo & Vai & Livro & Jardim & Gato & Mochila \\
\hline FADA & Chave & Faca & Cadeira & Foca & Olho & Caneta & Camiseta & Lousa \\
\hline
\end{tabular}

obtiveram resultados bastante superiores às dos grupos controle na tarefa de escrita. No momento de pré-teste, as produções escritas da totalidade das crianças não tinham relação com a oralidade e a composição interna das palavras era aleatória. No momento de pós-teste, as crianças dos grupos experimentais codificaram adequadamente segmentos das palavras, usando grafemas corretos para representar os seus fonemas constituintes.

Observaram-se também resultados superiores por parte das crianças dos grupos experimentais na tarefa de leitura no momento de pós-teste, o que sugere que as atividades de treino de escritas inventadas potenciaram a aquisição do princípio alfabético e a transferência de novos conhecimentos metalinguísticos para a habilidade de leitura. Esse padrão evolutivo foi notório nos grupos experimentais de uma forma geral, independentemente do país de proveniência das crianças.

Assim, o presente trabalho corrobora as pesquisas anteriores efetuadas no Canadá e em Portugal (Alves Martins et al., 2013, 2015; Alves Martins et al., 2014; Alves Martins \& Silva, 2006; Ouellette \& Sénéchal, 2008a, b; Ouellette, Sénéchal, \& Haley, 2013). Essas investigações experimentais destacaram os efeitos benéficos dos programas de treino de escritas inventadas para o desenvolvimento do pensamento metalinguístico e da escrita de crianças pré-escolares.

A eficácia do programa apresentado no presente estudo poderá ser explicada pela intervenção do adulto 
ao longo do treino que, a partir da mediação das interações entre as crianças, induziu o desenvolvimento do pensamento metalinguístico, encorajando-as a refletir explicitamente sobre as letras e os sons das palavras. Essa reflexão facilita a compreensão da estrutura alfabética da linguagem escrita, já que a mobilização de letras nas atividades de escrita inventada constitui um suporte concreto para a compreensão de unidades abstratas como os fonemas (Stahl \& Murray, 1998). Adams (1998) refere a esse propósito que "a evidência de que as atividades de escrita inventada desenvolvem simultaneamente a consciência fonêmica e promovem a compreensão do princípio alfabético é extremamente promissora, especialmente dadas as dificuldades que as crianças revelam ter na aquisição desta compreensão através de outros métodos de ensino" (p. 387).

A apresentação da forma correta da palavra foi um suporte essencial para que o adulto pudesse levar as crianças a interrogar-se sobre as diferenças entre a escrita produzida e a outra escrita, conduzindo-as assim, não a uma simples cópia, mas a uma compreensão mais aprofundada da natureza das relações entre os segmentos orais das palavras e os seus grafemas correspondentes. Com efeito, as escritas precoces são, de acordo com diversos pesquisadores, um fator importante para a aprendizagem, pois implicam uma reflexão que vai além de simples processos de cópia ou memorização (Mann, 1993; McBride-Chang, 1998; Ouellette \& Sénéchal, 2008a; b; Tolchinsky, 2005; Treiman, 1998).

A transferência de conhecimentos de escrita para habilidades de leitura corrobora os trabalhos de pesquisadores como Ehri (1997) e Rieben, Saada-Robert e Moro (1997), que defendem a existência de mecanismos comuns à leitura e à escrita e que enfatizam a interdependência na aquisição de ambas as habilidades. Também Treiman (1998) e Bowman e Treiman (2002) consideram que a escrita de palavras nos anos pré-escolares, ao encorajar as crianças a relacionar os grafemas com os fonemas, pode permitir o desenvolvimento de habilidades que poderão ser transferidas para a leitura.

Segundo Ouellette e Sénéchal (2008b), as atividades de escrita inventada levam as crianças a usar procedimentos analíticos que permitem a integração de informações fonológicas e alfabéticas em representações lexicais que, à medida que se tornam mais sofisticadas, potencializam a aprendizagem da leitura. Também Frith (1985) e Mann (1993) consideram que essas atividades permitem a aquisição de procedimentos que podem ser transferidos para a leitura de palavras.
Com efeito, a análise oral das palavras e o mapeamento do discurso oral na escrita permite a integração de representações fonológicas e ortográficas (Perfetti \& Hart, 2002; Share, 1995) essenciais para o processo de alfabetização.

Assim, esta pesquisa oferece uma contribuição importante, demonstrando a eficácia da metodologia adotada nesse tipo de programas de intervenção para a alfabetização inicial de crianças falantes de duas variantes do português: europeu e brasileiro.

\section{Considerações Finais}

Em suma, os dados do presente estudo fornecem fundamentação empírica pertinente para que se possa diversificar o projeto pedagógico na Educação Infantil, incluindo atividades de escrita inventada com vista ao sucesso na alfabetização inicial das crianças antes do ingresso no Ensino Fundamental. Assim, recomenda-se que, em estudos futuros, seja experimentada a aplicação desses programas de treino pelo próprio professor dentro do horário letivo e sem intervenção de um pesquisador.

Como limitações a esta pesquisa, considera-se que a dimensão da amostra é reduzida e que deveria incluir um maior número de participantes, não só em cada país, como ainda nos diferentes tipos de escola. Adicionalmente, será interessante analisar os recursos do ambiente familiar e as práticas de alfabetização presentes na vida das crianças fora do contexto escolar.

\section{Referências}

Adams, M. (1998). Beginning to read: Thinking and learning about print. Cambridge, MA: MIT Press.

Alves Martins, M., Albuquerque, A., Salvador, L., \& Silva, C. (2013). The impact of invented spelling on early spelling and reading. Journal of Writing Research, 5, 215-237. doi: 10.17239/jowr-2013.05.02.3.

Alves Martins, M., Albuquerque, A., Salvador, L., \& Silva, C. (2015). Escrita inventada e aquisição da leitura em crianças de idade pré-escolar. Psicologia: Teoria e Pesquisa, 31(2), 137-144. doi: 10.1590/0102-37722015021639137144.

Alves Martins, M., Salvador, L., Albuquerque, A., \& Silva, C. (2014). Invented spelling activities in small groups and early spelling and reading. Educational Psychology, 0, 1-15. doi: 10.1080/01443410.2014.950947. 
Alves Martins, M., \& Silva, C. (2006a). The impact of invented spelling on phonemic awareness. Learning and Instruction, 16, 41-56. doi: 10.1016/j. learninstruc.2005.12.005.

Alves Martins, M., \& Silva, C. (2006b). Phonological abilities and writing among Portuguese preschool children. European Journal of Psychology of Education, 21, 163-182. doi: 10.1007/BF03173575.

Bowman, M., \& Treiman, R. (2002). Relating print and speech: The effects of letter names and word position on reading and spelling performance. Journal of Experimental Child Psychology, 82, 305-340.

Boyer, N., \& Ehri, L. C. (2011). Contribution of phonemic segmentation instruction with letters and articulation pictures to word reading and spelling in beginners. Scientific Studies of Reading, 15(5), 440470. doi: 10.1080/10888438.2010.520778.

Byrne, B. (1998). The foundations of literacy. Hove, UK: Psychology Press.

Cardoso-Martins, C. (1995). Sensitivity to rhymes, syllables and phonemes in literacy acquisition in Portuguese. Reading Research Quarterly, 30, 808-828.

Cardoso-Martins, C., Mesquita, T. C. L., \& Ehri, L. C. (2011). Letter names and phonological awareness help children to learn letter-sound relations. Journal of Experimental Child Psychology, 109(1), 25-38. doi: 10.1016/j.jecp.2010.12.006.

Cardoso-Martins, C., Resende, S. M., \& Rodrigues, L. A. (2002). Letter name knowledge and the ability to learn to read by processing letter-phoneme relations in words: Evidence from Brazilian Portuguese-speaking children. Reading and Writing: An Interdisciplinary Journal, 15(3), 409-432. doi: 10.1023/A:1015213514722.

Castiglioni-Spalten, M. L., \& Ehri, L. C. (2003). Phonemic awareness instruction: Contribution of articulatory segmentation to novice beginners' reading and spelling. Scientific Studies of Reading, 7(1), 25-52. doi: 10.1207/S1532799XSSR0701_03.

Chomsky, C. (1970). Reading, writing and phonology. Harvard Educational Review, 40, 287-309. Recuperado de http://hepg.org/her-home/issues/ harvard-educational-review-volume-40,-issue-2/ herarticle/_1035

Cuesta, P. V., \& Luz, M. A (1971). Gramática da Lingua Portuguesa. Lisboa: Edições70.
Duarte, I. (2000). Lingua Portuguesa - Instrumentos de análise. Lisboa: U. Aberta.

Dehaene, S. (2012). Os neurônios da leitura: Como a ciência explica a nossa capacidade de ler. Porto Alegre, RS: Penso.

Ehri, L. C. (1991). The development of reading and spelling in children: An overview. In M. Snowling \& M. Thomson (Eds.), Dyslexia: Integrating theory and practice (pp. 63-94). London: British Dyslexia Association.

Ehri, L. C. (1997). Learning to read and learning to spell are one and the same, almost. Em C. Perfetti, L. Rieben, \& M. Fayol (Eds.), Learning to spell - Research, theory and practice across languages (pp. 237-269). New Jersey: LEA.

Ehri, L. C. (2014). Orthographic mapping in the acquisition of sight word reading, spelling memory and vocabulary learning. Scientific Studies of Reading, 18(1), 5-21. doi: 10.1080/10888438.2013.819356.

Ferreiro, E., \& Teberosky, A. (1979). Los sistemas de escritura en el desarrollo del niño. México: Siglo XXI.

Frith, U. (1985). Beneath the surface of developmental dyslexia. Em K. E. Patterson, J. C. Marshall \& M. Coltheart (Eds.), Surface dyslexia: Cognitive and neuropsychological studies of phonological reading (pp. 301-330), Hillsdale, NJ: Lawrence Erlbaum.

Gombert, E. (1990). Le développement métalinguistique. Paris: P.U.F.

Mann, V. A. (1993). Phoneme awareness and future reading ability. Journal of Learning Disabilities, 26, 259-269. doi: 10.1177/002221949302600406.

Mann, V. A., \& Foy, J. G. (2003). Speech development, phonological awareness and letter knowledge in preschool children. Annals of Dyslexia, 53, 149-173. doi: 10.1007/s11881-003-0008-2.

Mateus, M. H. M., Brito, A. M., Duarte, I., Faria, I. H., Frota, S., Matos, G., Oliveira, F., Vigário, M., \& Villalva, A. (2003). Gramática da Lingua Portuguesa. Lisboa: Editorial Caminho.

McBride-Chang, C. (1998). The development of invented spelling. Early Education and Development, 9, 147-160. doi: 10.1207/s15566935eed0902_3.

Moojen, S., Lamprecht, R., Santos, R. M., Freitas, G. M., Brodacz, R., Siqueira, M., Costa, A. C., \& Guarda, E. (2003). CONFIAS - Consciência Fonológica: 
Instrumento de Avaliação Seqüencial. São Paulo: Casa do Psicólogo.

Morais, J. (2013). Criar leitores - para professores e educadores. São Paulo: Manole.

Nunes, T., \& Bryant, P. (2009). Children's reading and spelling: Beyond the first steps. Oxford: Wiley-Blackwell.

Ouellette, G., \& Sénéchal, M. (2008a). A window into early literacy: Exploring the cognitive and linguistic underpinnings of invented spelling. Scientific Studies of Reading, 12(2), 195- 219. doi: 10.1080/10888430801917324.

Ouellette, G. \& Sénéchal, M. (2008b). Pathways to literacy: A study of invented spelling and its role in learning to read. Child Development, 79(4), 899-913. doi: 10.1111/j.1467-8624.2008.01166.x.

Ouellette, G., Sénéchal, M., \& Haley, A. (2013). Guiding children's invented spellings: A gateway into literacy learning. Journal of Experimental Education, 81(2), 261-279. doi: 10.1080/00220973.2012.699903.

Perfetti, C. A., \& Hart, L. (2002). The lexical quality hypothesis. Em L. Verhoeven, C. Elbro, \& P. Reitsma (Eds.), Precursors of functional literacy (pp.189-213). Philadelphia, PA: John Benjamins.

Pollo, T. C., Kessler, B., \& Treiman, R. (2005). Vowels, syllables and letters names: Differences of young children's spelling in English and Portuguese. Journal of Experimental Child Psychology, 92, 161-181.

Pollo, T. C., Treiman, R., \& Kessler, B. (2008). Three perspectives on spelling development. Em E. J. Grigorenko \& A. Naples (Eds.), Single-word read: Cognitive behavioral and biological perspectives (pp. 175189). Mahwah, NJ: Erlbaum.

Raven, J., Raven, J. C., \& Court, J. H. (1998). Manual for Raven's progressive matrices and vocabulary scale. Section I. General overview. Oxford: Oxford Psychologists Press.

Read,C. (1971). Pre-school children's knowledge of English phonology. Harvard Educational Review, 41, 1-34. Recuperado de http://hepg.org/her-home/issues/ harvard-educational-review-volume-41,-issue-1/ herarticle/_1011

Rieben, L., Saada-Robert, M., \& Moro, C. (1997). Word search strategies and stages of word recognition. Learning and Instruction, 7(2), 137-159. doi: 10.1016/ s0959-4752(96)00014-X.
Sénéchal, M., LeFevre, J. A., Smith-Chant, B. L., \& Colton, K. V. (2001). On refining theoretical models of emergent literacy: The role of empirical evidence. Journal of School Psychology, 95, 231-254. doi: 10.1016/S0022-4405(01)00081-4.

Sénéchal, M., Ouellette, G., Pagan, S., \& Lever, R. (2012). The role of invented spelling on learning to read in low-phoneme awareness kindergartners: A randomized-control-trial study. Reading and Writing, 25, 917-934. doi: 10.1007/s11145-011-9310-2.

Share, D. L. (1995). Phonological coding and self-teaching: Sine qua non of reading acquisition. Cognition, 55, 151-2218.

Silva, C. (2008). Bateria de provas fonológicas. Lisboa: ISPA.

Silva, C., \& Alves Martins, M. (2002). Phonological skills and writing of presyllabic children. Reading Research Quarterly, 37, 466-483. doi: 10.1598/RRQ.37.4.6.

Snow, C. (1991). The theoretical basis for relationships between language and literacy in development. Journal of Research in Childhood Education, 6, 5-10. doi: 10.1080/02568549109594817.

Stahl, S., \& Murray, B. (1998). Issues involved in defining phonological awareness and its relation to early reading. Em J. L. Metsala \& L. C. Ehri (Eds.), Word recognition in beginning literacy (pp. 65-85). London: Erlbaum.

Teale, W. H., \& Sulzby, E. (1986). Emergent literacy: Writing and reading. Norwood, NJ: Ablex.

Tolchinsky, L. (2005). The emergence of writing. Em C. MacArthur, S. Graham \& J. Fitzgerald (Eds.), Handbook of writing research (pp. 83-96). New York: Guilford.

Treiman, R. (1998). Why spelling? The benefits of incorporating spelling into beginning reading instruction. Em J. L. Metsala \& L.C. Ehri (Eds.), Word recognition in beginning literacy (pp. 289-313). Mahwah, NJ: Erlbaum.

Treiman, R. (2004). Phonology and spelling. Em P. Bryant \& T. Nunes (Eds.), Handbook of children's literacy (pp. 31-42). Dordrecht, the Netherlands: Kluwer.

Treiman, R. \& Kessler, B. (2003). The role of letter names in the acquisition of literacy. Em R. Kail (Eds.), Advances in child development and behavior (pp. 105-135). San Diego: Academic Press. 
Treiman, R., Tincoff, R., Rodriguez, K., Mozaki, A.,

Recebido em: 22/10/2015 \& Francis, D. (1998). The foundation of literacy: Learning the sounds of the letters. Child Development, 69, 1524-1540.

Reformulado em: 30/03/2016; 16/04/2016

Aprovado em: 24/05/2016

Nota das autoras:

Agradecemos à Fundação para a Ciência e Tecnologia (FCT) pelo apoio financeiro concedido ao primeiro autor, que possibilitou a execução desta pesquisa (bolsa de doutorado com a referência SFRH/ BD/112133/2015).

Sobre as autoras:

Ana Albuquerque é doutoranda em Psicologia no ISPA - Instituto Universitário, Lisboa, Portugal, com estágio no Instituto de Psicologia da Universidade de São Paulo, Brasil, e bolsista pela Fundação para a Ciência e Tecnologia. Colabora no Centro de Investigação em Educação (ISPA-IU), tem experiência em projetos científicos e participação em eventos e publicações internacionais. Os seus principais temas de pesquisa são as habilidades metalinguísticas e de alfabetização inicial.

E-mail: aalbuquerque@ispa.pt

Margarida Alves Martins é professora catedrática de Psicologia da Educação no ISPA - Instituto Universitário, Lisboa, Portugal, doutorada em Psicologia pela Universidade de Coimbra, Portugal e em Ciências da Educação pela Universidade René Descartes - Paris V, França e coordena o CIE - Centro de Investigação em Educação (ISPA-IU). Os seus interesses de investigação são na área da alfabetização inicial.

E-mail:mmartins@ispa.pt

Contato com as autoras:

CIE - Centro de Investigação em Educação, ISPA - Instituto Universitário

Rua Jardim do Tabaco, 34, 1149-041

Lisboa, Portugal 